\title{
Efeito de diferentes doses de nandrolona associado ao treinamento de força sobre o perfil fenotípico e área de secção transversa do músculo de ratos
}

CDD. 20.ed. 796.073

\author{
Rozangela VERLENGIA* \\ Jonato PRESTES*** \\ Ricardo Yukio ASANO** \\ Wagner José da SILVA* \\ Gerson Eduardo Rocha de CAMPOS* \\ Cláudia Regina CAVAGLIERI*** \\ Rodrigo Duarte de SOUZA* \\ Sérgio Ricardo BOFF" \\ Silvia Cristina Crepaldi ALVES* \\ *Faculdade de \\ Ciências da Saúde \\ Universidade Metodista \\ de Piracicaba. \\ ** Universidade \\ Católica de Brasília. \\ ***Faculdade de \\ Educação Física, \\ Universidade Estadual \\ de Campinas.
}

\section{Resumo}

0 presente estudo avaliou a influência de diferentes doses de decanoato de nandrolona (DN) associado ao Treinamento de Força (TF) sobre o fenótipo de fibras e área de secção transversa (AST) do músculo extensor longo dos dedos (EDL) em ratos "Wistar". Os animais foram divididos em sete grupos: controle (GC) e grupos de acordo com a concentração de $D N(0,1,1,2,5$, 10 e $20 \mathrm{mg} / \mathrm{kg})$ administrada intramuscular 3 vezes/semana. 0 TF consistiu de saltos em meio líquido (carga 50-70\% do peso corporal) 3x/semana, durante cinco semanas. A associação do TF e DN promoveu ação modulatória sobre os tipos de fibras. Houve hipertrofia das fibras de contração rápida (tipo II) em comparação com as fibras de contração lenta (tipo I). Em conclusão, apesar da associação do TF com DN aumentar a AST muscular e alterar o fenótipo das fibras, não houve efeito gradual das doses mais altas.

PalAVRAS-ChAVE: Treinamento de força; Esteróide anabolizante; Fibras musculares; Área de secção transversa muscular.

\section{Introdução}

O treinamento físico associado com as respostas hormonais pode contribuir para modificar a característica fenotípica das fibras musculares desencadeando uma série de eventos intracelulares, relacionados a fatores metabólicos e mecânicos ${ }^{1-4}$.

De forma geral, repetidas sessões de treinamento de força realizados em ratos ou humanos, promovem adaptaçôes nas fibras musculares por meio de alteraçôes do conteúdo protéico intramuscular (síntese protéica), resultando no aumento da área de secção transversa (AST) da musculatura esquelética e adaptações no fenótipo das fibras (transição das fibras) ${ }^{5-7}$.

A testosterona é um hormônio esteróide produzido a partir do colesterol, e tem sido extensivamente investigado em relação à indução das adaptações nas fibras musculares, por apresentar uma importante função anabólica ${ }^{8-10}$. Por esta razão, passou a ser utilizada e comercializada na forma de esteróide anabólico androgênico (EAA) sintético ${ }^{11}$, com aplicação clínica médica ${ }^{11-13}$, bem como para o aumento do desempenho físico, principalmente nas modalidades que necessitam de alta produção de força e potência muscular ${ }^{14}$. No entanto, vale ressaltar que a utilização dos EAA para essa finalidade é proibida pelo Comitê Olímpico Internacional (COI) ${ }^{15-16}$.

Farmacologicamente, a maioria dos EAA sintéticos disponíveis se origina da testosterona, dentre estes o decanoato de nandrolona $(\mathrm{DN})^{17}$. O DN se 
assemelha à testosterona, porém possui maior efeito anabólico, decorrente da substituição do átomo de hidrogênio no grupo metil C19 da testosterona, que favorece a relação anabólica/androgênica ${ }^{11}$.

Nos últimos anos, tem se investigado os efeitos da utilização de EAA isolados e/ou associados ao treinamento físico sobre o perfil fenotípico das fibras musculares e AST ${ }^{18-19}$. Nesse sentido, KonisHi et al. ${ }^{19}$ verificaram que após seis semanas de administração diária de DN (1 mg/kg), houve aumento na distribuição de fibras tipo IIB e AST do músculo extensor longo dos dedos (EDL) de ratos. Adicionalmente, FERRY et al. ${ }^{18}$ ao analisarem o efeito do uso de DN de forma isolada ou associada ao exercício sobre o processo de

\section{Método}

\section{Animais}

Foram utilizados ratos machos da linhagem "Wistar" ("Rattus novergius var. albinus Rodentia, Mammalia"), com dois meses de idade e peso médio de 281,16 $\pm 21,24 \mathrm{~g}$, obtidos no Biotério Central da Universidade Metodista de Piracicaba - UNIMEP. Os animais foram separados em gaiolas coletivas (cinco animais em cada) e receberam ração e água "ad libitum" (Purina, Descalvado, São Paulo, Brasil). A temperatura da sala foi controlada a $23 \pm 2{ }^{\circ} \mathrm{C}$, com ciclo claro-escuro de 12/12 h. Antes de iniciar o período experimental, os animais permaneceram por $48 \mathrm{~h}$ em adaptação às condições do biotério de pesquisa. Todos os experimentos envolvendo os animais foram realizados em concordância com as resoluções brasileiras específicas da Bioética em Experimentos com Animais (projeto de lei n. 93/08, aprovado em 9 de setembro de 2008). O experimento foi aprovado pelo Comitê de Ética em Pesquisa para animais experimentais do Instituto de Ciências Biomédicas/USP ( $n^{\circ}$ 49).

\section{Desenho experimental}

Os animais foram separados aleatoriamente em sete grupos de cinco animais. Seis destes grupos receberam injeçōes intramusculares de $\mathrm{DN}$, três vezes por semana: grupo $1(\mathrm{GN} 1)-0,1 \mathrm{mg} / \mathrm{kg}$ de $\mathrm{DN}$; grupo 2 (GN2) - 1,0 mg/kg de DN; grupo 3 (GN3) - 2,0 $\mathrm{mg} / \mathrm{kg}$ de DN; grupo 4 (GN4) $-5,0 \mathrm{mg} / \mathrm{kg}$ de $\mathrm{DN}$; grupo 5 (GN5) - 10,0 mg/kg; e grupo 6 (GN6) - $20 \mathrm{mg} / \mathrm{kg}$. Os animais do grupo controle regeneração muscular, concluíram que as combinaçōes de ambos os procedimentos potencializaram a síntese protéica nos músculos EDL e sóleo de ratos.

No entanto, a relação dos efeitos da dose-resposta da administração de DN associada ao treinamento de força sobre o músculo esquelético ainda precisa ser determinada. Diante do exposto, o objetivo do presente estudo foi analisar o efeito de diferentes doses de DN associado ao treinamento de força sobre o perfil fenotípico das fibras musculares e AST do músculo EDL em ratos. A hipótese inicial era de que o treinamento de força associado com maior dose de DN produziria superior aumento da AST, bem como alteração fenotípica das fibras musculares.

(GC) receberam injeções intramusculares de veículo $(0,2 \mathrm{~mL}$ de propilenoglicol), três vezes por semana. Antes de cada aplicação de DN os animais eram pesados e a concentração de $\mathrm{DN}$ era ajustada pelo peso. A administração de DN foi realizada após o treinamento, nos músculos do quadríceps da coxa, de forma alternada entre as patas. $\mathrm{O}$ volume das injeções foi $0,1 \mathrm{~mL} / 200 \mathrm{~g}$ de peso/animal ${ }^{20}$. Os espectros das doses usadas variam de baixas doses (reposição hormonal) a altas doses (abuso de EAA em humanos) e doses que demonstraram ter efeito sobre o músculo e performance física de ratos ${ }^{21-25}$.

\section{Treinamento de força}

Inicialmente os animais foram adaptados ao meio líquido durante uma semana, e na semana seguinte ao meio líquido e treino para reduzir o estresse. A adaptação consistiu de sessões de saltos na água $(30 \pm 2$ ${ }^{\circ} \mathrm{C}$ ) com carga presa ao peito ( $50 \%$ do peso corporal) uma vez ao dia por cinco dias, com aumento gradual no número de séries (dois para quatro) e de saltos (cinco para 10). Todas as sessões de adaptação foram realizadas pela manhã (entre 8 e $12 \mathrm{~h})^{22}$. O propósito da adaptação foi para reduzir o estresse do animal, quando da realização do protocolo experimental.

Posteriormente, os animais foram submetidos ao protocolo de treinamento de força por cinco semanas $(25 \text { sessóes })^{26}$. O protocolo consistiu de quatro séries de 10 saltos cada com intervalo de 30 s. Nas duas primeiras semanas a carga foi de $50 \%$ do peso corporal; na terceira e quarta $60 \%$ do peso corporal; na quinta semana $70 \%$ do peso corporal. 


\section{Obtenção da amostra}

As amostras do músculo EDL foram obtidas $48 \mathrm{~h}$ após a última sessão de treino (tempo para minimizar o efeito da resposta aguda), sendo fixadas em blocos de madeira e mantidas em nitrogênio líquido para posterior análise histológica. Os cortes transversais do músculo foram obtidos por meio de criostato (Mícron $\left.{ }^{\circledR} \mathrm{HM} 505\right)$ à $24^{\circ} \mathrm{C}$ na espessura de $12 \mu \mathrm{m}$.

\section{Determinação das isoformas da miosina de cadeia pesada (MHC) no músculo EDL}

A determinação da distribuição dos tipos de fibras foi realizada por meio da análise histoquímica da atividade do trifosfato de adenosina miofibrilar (mATPase) em incubações com pH de 4,30, 4,55 e 10,60 seguindo as recomendaçoós de BroOKe e KaISER ${ }^{27}$ e STARON e PeTTE $^{28}$. Para a contagem do número e identificação das fibras foram feitas imagens de três campos aleatórios de cada corte nos $\mathrm{pH}$ de 4,30, 4,55 e 10,60, mantendo a mesma região do músculo. Para tal, foi utilizado um microscópio Nikon acoplado a uma câmara fotográfica. As imagens capturadas foram transferidas para o computador e analisadas por meio do sistema de análise de imagens Image-Pro Plus 4.0 - Media Cybernetics.

\section{Determinação da AST da fibra muscular do EDL}

A mensuração da AST das fibras (análise morfométrica) foi realizada a partir de imagens obtidas em três campos aleatórios feitos nos $\mathrm{pHs}$ de 4,30, 4,55 e 10,6, sendo analisadas pelo menos 50 fibras de cada tipo por meio do "software" Image-Pro Plus 4.0 - Media Cybernetics. A AST das fibras musculares foi obtida em micrômetros quadrados $\left(\mu \mathrm{m}^{2}\right)$.

\section{Análise estatística}

Todos os dados estão apresentados em média e desvio padrão da média (DP). Para testar a normalidade dos dados foi utilizado o teste de Kolmogorov-Smirnov e para homocedasticidade o critério de Bartlett. Todas as variáveis analisadas apresentaram distribuição normal e homocedasticidade. Sendo assim, foi utilizada a análise de variância multifatorial (ANOVA) seguido do pós-teste de Tukey-Kraemer. Em todos os cálculos foi fixado um nível crítico de $5 \%(\mathrm{p}<0,05)$. O "software" utilizado em todos os testes estatísticos foi o SPSS ${ }^{\circledR}$ versão 10.

\section{Resultados}

Os resultados referentes à distribuição dos tipos de fibras após cinco semanas de treinamento de força associado com diferentes doses de DN estão expressos na TABELA 1. Foi observado aumento significativo $(\mathrm{p}<0,05)$ nas fibras do tipo IIAC, IIA, IIAD, IID, IIDB e IIB em todos os grupos, independente da dosagem administrada. Houve redução significativa $(\mathrm{p}<0,05)$ das fibras do tipo I somente nos grupos que utilizaram dosagens a partir de $2 \mathrm{mg} / \mathrm{kg}$ (GN3, GN4, GN5 e GN6) quando comparado ao grupo controle. Nos demais tipos de fibras houve aumento significativo $(\mathrm{p}<0,05)$ comparado ao grupo controle, independente da dosagem administrada, com exceção das fibras do tipo IC e IIC, que demonstraram aumento a partir de $1 \mathrm{mg} / \mathrm{kg}$ e $2 \mathrm{mg} / \mathrm{kg}$, respectivamente. As fibras híbridas IIAC e IIAD foram as que apresentaram maior magnitude de aumento percentual em relação ao grupo controle. Não houve diferença significativa ( $\mathrm{p}>0,05)$ entre as diferentes doses de DN.

Os valores absolutos da AST estão expressos na TABELA 2. Houve diminuição significativa $(\mathrm{p}<0,05)$ da AST da fibra IC em todos os grupos experimentais em comparação ao grupo controle, independente da dosagem administrada. Na fibra IIB foi observado aumento significativo $(\mathrm{p}<0,05)$ da AST em todas as doses. Nas fibras tipo I houve aumento da AST nos grupos que receberam dosagens de 2,5 e 20 $\mathrm{mg} / \mathrm{kg}$. Nas fibras tipo IIC foi observado aumento significativo $(\mathrm{p}<0,05)$ na AST somente nos grupos que receberam 5 e $20 \mathrm{mg} / \mathrm{kg}$. Para os demais tipos de fibras não foram observadas alterações estatisticamente significativas $(p>0,05)$, bem como não houve diferença entre as doses de ND. 
TABELA 1 - Média e desvio padrão da porcentagem de distribuição dos tipos de fibras segundo a cadeia pesada de miosina do músculo extensor longo dos dedos de ratos após treinamento de força associado às diferentes doses de decanoato de nandrolona.

$G C=$ grupo controle; $\mathrm{GN}=$ grupo nandrolona. * = diferença significativa em relação ao grupo controle $(p<0,05)$.

\begin{tabular}{|c|c|c|c|c|c|c|c|}
\hline Fibras & $\begin{array}{c}\text { GC Controle } \\
(\%)\end{array}$ & $\begin{array}{c}\text { GN1 } 0,1 \mathrm{mg} / \mathrm{kg} \\
(\%)\end{array}$ & $\begin{array}{c}\text { GN2 } 1 \mathrm{mg} / \mathrm{kg} \\
(\%)\end{array}$ & $\begin{array}{c}\text { GN3 } 2 \mathrm{mg} / \mathrm{kg} \\
(\%)\end{array}$ & $\begin{array}{c}\text { GN4 } 5 \mathrm{mg} / \mathrm{kg} \\
(\%)\end{array}$ & $\begin{array}{c}\text { GN5 } 10 \mathrm{mg} / \mathrm{kg} \\
(\%)\end{array}$ & $\begin{array}{c}\text { GN6 } 20 \mathrm{mg} / \mathrm{kg} \\
(\%)\end{array}$ \\
\hline I & $4,14(1,22)$ & $4,03(0,81)$ & $4,02(0,71)$ & $3,45(0,76)^{*}$ & $3,17(1,26)^{*}$ & $3,89(1,41)^{*}$ & $3,18(0,81)^{*}$ \\
\hline IIC & $0,25(0,71)$ & $0,34(1,58)$ & $0,34(0,71)$ & $0,70(0,76)^{*}$ & $0,25(1,54)$ & $0,41(1,00)^{*}$ & $0,50(0,81)^{*}$ \\
\hline IIAC & $1,06(1,58)$ & $3,53(1,58)^{*}$ & $3,5(1,34)^{*}$ & $3,27(1,85)^{*}$ & $2,83(0,63)^{*}$ & $3,15(1,78)^{*}$ & $2,34(0,81)^{*}$ \\
\hline IIAD & $4,00(1,48)$ & $9,41(1,58)^{*}$ & $9,83(1,41)^{*}$ & $10,80(1,45)^{*}$ & $11,75(1,26)^{*}$ & $10,52(2,23)^{*}$ & $11,54(1,60)^{*}$ \\
\hline IID & $27,94(1,22)$ & $40,17(1,22)^{*}$ & $39,74(1,87)^{*}$ & $40,40(1,60)^{*}$ & $39,50(2,28)^{*}$ & $38,36(2,00)^{*}$ & $38,80(0,81)^{*}$ \\
\hline IIBD & $14,80(1,22)$ & $21,01(1,58)^{*}$ & $21,11(1,22)^{*}$ & $22,83(0,93)^{*}$ & $21,92(1,89)^{*}$ & $19,47(1,41)^{*}$ & $22,99(1,41)^{*}$ \\
\hline IIB & $31,44(1,58)$ & $15,04(0,70)^{*}$ & $15,05(1,22)^{*}$ & $13,50(2,00)^{*}$ & $14,42(0,63)^{*}$ & $18,23(1,00)^{*}$ & $15,38(2,82)^{*}$ \\
\hline
\end{tabular}

TABELA 2 - Média e desvio padrão da AST $\left(e m \mu^{2}\right.$ ) dos diferentes tipos de fibras do músculo EDL de ratos após treinamento de força associado às diferentes doses de DN.

\begin{tabular}{|c|c|c|c|c|c|c|c|c|}
\hline \multirow[t]{10}{*}{$\begin{array}{l}{ }^{*}=\text { diferença significativa } \\
\text { em relação ao grupo } \\
\text { controle }(p \leq 0,05) .\end{array}$} & Fibras & GC Controle & $\begin{array}{c}\text { GN1 } \\
0,1 \mathrm{mg} / \mathrm{kg}\end{array}$ & $\begin{array}{c}\text { GN2 } \\
1 \mathrm{mg} / \mathrm{kg}\end{array}$ & $\begin{array}{c}\mathrm{GN} 3 \\
2 \mathrm{mg} / \mathrm{kg}\end{array}$ & $\begin{array}{c}\text { GN4 } \\
5 \mathrm{mg} / \mathrm{kg}\end{array}$ & $\begin{array}{c}\text { GN5 } \\
10 \mathrm{mg} / \mathrm{kg}\end{array}$ & $\begin{array}{c}\text { GN6 } \\
20 \mathrm{mg} / \mathrm{kg}\end{array}$ \\
\hline & I & $1467(230)$ & $1478(310)$ & $1476(320)$ & $1537(222)^{*}$ & $1542(320)^{*}$ & $1498(222)$ & $1548(346)^{*}$ \\
\hline & IC & $1440(234)$ & $1309(3248)^{*}$ & $1298(324)^{*}$ & $1322(234)^{*}$ & $1312(160)^{*}$ & $1348(273)^{*}$ & $1350(412)^{*}$ \\
\hline & IIC & $1269(285)$ & $1298(248)$ & $1282(349)$ & $1269(285)$ & $1310(204)^{*}$ & $1298(385)$ & $1305(340)^{*}$ \\
\hline & IIAC & $1123(312)$ & $1213(213)$ & $1215(385)$ & $1285(285)$ & $1232(248)$ & $1265(231)$ & $1272(238)$ \\
\hline & IIA & $1625(412)$ & $1638(212)$ & $1611(424)$ & $1650(394)$ & $1683(412)$ & $1690(309)$ & $1679(394)$ \\
\hline & IIAD & $1770(320)$ & $1788(320)$ & $1798(380)$ & $1785(309)$ & $1799(230)$ & $1785(485)$ & $1793(380)$ \\
\hline & IID & $1953(251)$ & 1990 (278) & $1989(238)$ & $1993(380)$ & $1985(241)$ & $1995(318)$ & $1989(254)$ \\
\hline & IIBD & $1342(248)$ & $1348(302)$ & $1342(203)$ & $1403(210)$ & $1358(320)$ & 1367 (273) & 1389 (309) \\
\hline & IIB & $2208(254)$ & $2405(310)^{*}$ & $2426(341)^{*}$ & $2602(254)^{*}$ & $2626(341)^{*}$ & $2640(254)^{*}$ & $2652(290)^{*}$ \\
\hline
\end{tabular}

\section{Discussão}

O objetivo do presente estudo foi analisar o efeito crônico de diferentes doses de DN associado ao treinamento de força sobre a AST do músculo EDL e perfil fenotípico das fibras musculares de ratos. Nossos dados indicaram um processo de transição fenotípica nos diferentes tipos de fibras musculares do músculo EDL, independente da dosagem administrada de DN, com maior impacto nas fibras híbridas do tipo IIAC e IIAD. Em relação a AST, houve um aumento nas fibras tipo IIB independente da dose de DN; por outro lado, nas fibras do tipo I ocorreu aumento a partir de $2 \mathrm{mg} / \mathrm{kg}$. Nas fibras híbridas foi observado aumento das fibras do tipo IIC e diminuição das fibras do tipo IC. Sendo assim, a associação do treinamento de força com DN alterou o perfil fenotípico das fibras musculares do EDL, sem efeito adicional da dose.
O DN pode interferir diretamente no processo adaptativo muscular, tanto de forma isolada quanto associada ao treinamento físico ${ }^{15,18-19,29-31}$. Nesse sentido, a ação desse hormônio pode induzir alterações fenotípicas das fibras musculares, dentre estas, a transição das fibras tipo I para tipo $\mathrm{II}^{30}$.

O presente estudo demonstrou que as alterações fenotípicas das fibras musculares ocorreram independente da dosagem de DN. Foi observada diminuição da porcentagem das fibras tipo I a partir da dose $2 \mathrm{mg} / \mathrm{kg}$. Em adição, houve aumento percentual das fibras tipo IC nos grupos GN2 $(1 \mathrm{mg} /$ $\mathrm{kg})$, GN3 (2 mg/kg), GN4 (5 mg/kg), GN5 (10 mg/ $\mathrm{kg})$ e GN6 $(20 \mathrm{mg} / \mathrm{kg})$, com aumento percentual também observado das fibras do tipo II (AC, A, $\mathrm{AD}, \mathrm{D}$ e $\mathrm{BD})$. 
Outros estudos demonstraram mudanças do perfil fenotípico das fibras musculares após o treinamento de força, resultando no aumento das fibras do tipo II, com predomínio das fibras do tipo IID e diminuição das fibras do tipo I, IIA e IIAD ${ }^{32-38}$. Deste modo, tal comportamento se confirmou no presente estudo, onde foi observado aumento de $-140 \%$ das fibras do tipo IID em comparação ao grupo controle, e diminuição de $-65 \%$ e $-8 \%$ das fibras IIA e I, respectivamente. Com exceção observada nos grupos GN2 e GN3, que não apresentaram alteração significativa no percentual das fibras tipo I. No entanto, houve um aumento expressivo no percentual das fibras tipo IIAD em todos os grupos experimentais (entre 220,7$333 \%$ ) comparados ao grupo controle.

Além da mudança do perfil fenotípico das fibras musculares, o treinamento anaeróbio pode modificar a composição corporal e, dependendo da intensidade e volume, pode desencadear também uma resposta hipertrófica das fibras musculares ${ }^{39}$. Nesse sentido, Camargo Filho et al. ${ }^{40}$ realizaram um estudo com administração de $5 \mathrm{mg} / \mathrm{kg}$ de DN (2x/semana) associado a 42 sessões treinamento de natação em ratos. Após nove semanas foram encontradas diferenças significativas na AST da fibra muscular do sóleo entre os grupos que realizaram o treinamento (tratados ou não com DN), sugerindo que os resultados obtidos se devem a realização do treinamento por si só. Entretanto, outros trabalhos mostraram os efeitos positivos dos EAA associado ao treinamento de força sobre a AST das fibras ${ }^{29,41}$.

No entanto, as alterações proporcionadas pelo uso de EAA sobre a AST das fibras musculares são divergentes no que diz respeito aos tipos de fibras que sofrem as adaptaçôes. Alguns autores demonstraram maior aumento nas fibras do tipo $\mathrm{I}^{42}$ e outros apontam aumento mais evidente nas fibras do tipo $\mathrm{II}^{43-44}$. Nessa linha, alguns estudos sugerem uma tendência de aumento mais pronunciado nas fibras tipo IID e IIB $^{30}$. Em partes, os resultados do presente estudo confirmam essa tendência, pois em todos os grupos experimentais houve hipertrofia das fibras tipo IIB na ordem de $\sim 11$ $12 \%$. Deste modo, as diferenças entre os resultados dos estudos podem estar associadas ao tipo de EAA utilizado, bem como ao protocolo de treinamento.
Outro objetivo do presente estudo foi analisar a relação dose-resposta referente à dosagem de DN e a sua influência na AST das fibras musculares. SinHA-Hikim et al. ${ }^{8}$ administraram doses semanais de 25, 50, 125, 300 e $600 \mathrm{mg}$ de testosterona por um período de 20 semanas em homens jovens saudáveis. Ao final deste período, os autores observaram aumento significativo na AST das fibras do tipo I (vasto lateral) nos grupos que receberam 300 e 600 mg por semana, e aumento das fibras do tipo II na dosagem de $600 \mathrm{mg}$ por semana. No entanto, não foi observada alteração do percentual das fibras do tipo I e II entre as dosagens de testosterona.

Em contrapartida, no presente estudo foi observado aumento da AST das fibras tipo I, IIC e IIB e diminuição nas fibras tipo IC. Por outro lado, nas fibras que apresentaram aumento significativo da AST, não foi encontrada qualquer relação entre diferentes dosagens de DN e hipertrofia das fibras musculares. Uma limitação do presente estudo é que o tempo de intervenção pode ter sido insuficiente para que possíveis adaptações significativas ocorressem nas fibras musculares em relação às diferentes dosagens de DN, uma vez que mudanças na AST das fibras musculares são percebidas depois de determinado período de treinamento crônico. Essas alteraçôes são caracterizadas, principalmente, pelo aumento na síntese de proteínas contráteis ${ }^{28,38,45}$. Por outro lado, o DN é um potente EAA, associado frequentemente com aumento do tamanho das fibras musculares ${ }^{17,19,24}$.

$\mathrm{O}$ uso de diferentes doses de DN associado ao treinamento de força promoveu aumento nas fibras do tipo IIAC e IIA e diminuição das fibras do tipo I, IIA e IIB, indicando uma ação modulatória sobre o perfil fenotípico do músculo EDL de ratos. Em adição, todas as dosagens avaliadas induziram a um aumento significativo da AST das fibras musculares do tipo IIB, o que é sugestivo de um processo de hipertrofia relacionado às fibras de contração rápida. Entretanto, não foi observada relação consistente entre o aumento da dosagem de DN e alterações pronunciadas no fenótipo das fibras musculares e AST, pelo menos no período analisado (cinco semanas) em ratos. 


\begin{abstract}
Effect of different doses of nandrolone associated with resistance training on muscle phenotypic profile and cross-sectional area of rats

The aim of the present study was to evaluate the influence of different doses of nandrolone decanoate (ND) associated with Strength Training (ST) on the phenotype of fibers and cross-sectional area (CSA) of the extensor digitorum longus (EDL) in Wistar rats. The animals were divided into seven groups: control (CG) and the groups according to the dose of ND administered $(0,1,1,2,5,10$ e $20 \mathrm{mg} / \mathrm{kg})$. The ST consisted of water jumping with loads of $50-70 \%$ of their body mass, three times per week during five weeks. The association of ST with ND promoted a modulatory role on the muscle fiber types. There was a hypertrophy of fast twitch fibers (type II) as compared with slow twitch (type I). In conclusion, although the association of ST with ND increased muscle CSA and modified fiber phenotype, there was no additional effect of higher doses.
\end{abstract}

KEY WORDS: Strength training; Anabolic steroid; Muscle fibers; Muscle cross-sectional area.

\title{
Referências
}

1. Pette D, Staron RS. Myosin isoforms, muscle fiber types and transitions. Microsc Res Tech. 2000;50:500-9.

2. Fluck M, Hoppeler H. Molecular basis of skeletal muscle plasticity-from gene to form and function. Rev Physiol Biochem Pharmacol. 2003;146:159-216.

3. Kraemer WJ, Ratamess NA. Fundamentals of resistance training: progression and exercise prescription. Med Sci Sports Exerc. 2004;36:674-88.

4. Spiering BA, Kraemer WJ, Anderson JM, et al. Resistance exercise biology: manipulation of resistance exercise programme variables determines the responses of cellular and molecular signalling pathways. Sports Med. 2008;38:527-40.

5. Pette D, Staron RS. Mammalian skeletal muscle fiber type transition. Int Rev Cytol. 1997;170:143-223.

6. Campos GER, Luecke TJ, Wendeln HK, et al. Muscular adaptations in response to three different resistance-training regimens: specificity of repetition maximum training zones. Eur J Appl Physiol. 2002;88:50-60.

7. Yamada AK, Verlengia R, Bueno Junior CR. Mechanotransduction pathways in skeletal muscle hypertrophy. Recept Signal Transduct Res. 2012;32:42-4.

8. Sinha-Hikim I, Artaza J, Woodhouse L, et al. Testosterone-induced increase in muscle size in healthy young men is associated with muscle fiber hypertrophy. Am J Physiol Endocrinol Metab. 2002;283:154-64.

9. Herbst KL, Bhasin S. Testosterone action on skeletal muscle. Curr Opin Clin Nutr Metab Care. 2004;7:271-7.

10. Vingren JL, Kraemer WJ, Ratamess NA, Anderson JM, Volek JS, Maresh CM. Testosterone physiology in resistance exercise and training: the up-stream regulatory elements. Sports Med. 2010;40:1037-53.

11. Shahidi NT. A review of chemistry, biological action and clinical applications of anabolic androgenic steroid. Clin Ther. 2001;23:1355-90.

12. Strawford A, Barbieri T, Neese R, et al. Effects of nandrolone decanoate therapy in borderline hypogonadal men with HIV-associated weight loss. J Acquir Immune Defic Syndr Hum Retrovirol. 1999;20:137-46.

13. Hartgens F, Kuipers H. Effects of androgenic-anabolic steroids in athletes. Sports Med. 2004;34:513-54.

14. Strauss RH, Yesalis CE. Anabolic steroids in the athlete. Annu Rev Med. 1991;42:449-57.

15. Silva PRP, Danielski R, Czepielewski MA. Esteróides anabolizantes no esporte. Rev Bras Med Esporte. 2002;8:235-43.

16. Graham MR, Davies B, Grace FM, Kicman A, Barker JS. Anabolic steroid use: patterns of use and detection of doping. Sports Med. 2008;38:505-24.

17. Kuhn CM. Anabolic steroids. Exerc Sport Sci Rev. 2002;57:411-34.

18. Ferry A, Noirez P, Page CL, Salah IB, Daegelen D, Rieu M. Effects of anabolic/androgenic steroids or regenerating skeletal muscle in the rat. Acta Physiol Scand. 1999;166:105-10.

19. Konishi M, Tokuhara N, Iwamoto S. The effect of anabolic steroid on the distribution of muscle fiber in rat hindlimb. Ital J Anat Embryol. 2001;106:175-83. 
20. Mirkhani H, Golbahar J, Shokri S. Chronic administration of nandrolone decanoate does not increasethe plasma homocysteine level of male rats. Basic Clin Pharmacol Toxicol. 2005;97:214-7.

21. Ferry A, Viganaud A, Noirez P, Bertucci W. Respective effects of anabolic/androgenic steroids and physical exercise on isometric contractile properties of regenerating skeletal muscles in the rat. Arch Physiol Biochem. 2000;108:257-61.

22. Gayan-Ramirez G, Rollier H, Vanderhoydonc F, Verhoeven G, Gosselink R, Decramer M. Nandrolone decanoate does not enhance training effects but increases IGF-I mRNA in rat diaphragm. J Appl Physiol. 2000;88:26-34.

23. Joumaa WH, Leoty C. Differential effects of nandrolone decanoate in fast and slow rat skeletal muscles. Med Sci Sports Exerc. 2001;33:397-403.

24. Kindlundh AM, Lindblom J, Bergström L, Nyberg F. The anabolic-androgenic steroid nandrolone induces alterations in the density of serotonergic 5HT18 and 5HT2 receptors in the male rat brain. Neuroscience. 2003;119:113-20.

25. Alsiç J, Birgner C, Bj̧̧rkblom L, et al. Impact of nandrolone decanoate on gene expression in endocrine systems related to the adverse effects of anabolic androgenic steroids. Basic Clin Pharmacol Toxicol. 2009;105:307-14.

26. Oliveira CAM, Rogatto GP, Luciano E. Effects of high intensity physical training on the leukocytes of diabetics rats. Rev Bras Med Esporte. 2002;8:219-24.

27. Brooke MH, Kaiser KK. Three "myosin ATPase" systems: the nature of their ph lability and sulfhydryl dependence. J Histochem Cytochem. 1970;18:670-2.

28. Staron RS, Pette D. The continnum of pure and hybrid myosin heavy chain the based fiber types in rat skeletal muscle. Histochem Cell Biol. 1993;100:149-53.

29. Kadi F, Eriksson A, Holmner S, Thornell LE. Effects of anabolic steroids on the muscle cells of strength-trained athletes. Med Sci Sports Exerc. 1999;31:1528-34.

30. Schroeder ET, Terk M, Sattler FR. Androgen therapy improves muscle mass and strength but not quality: results from two studies. Am J Physiol Endocrinol Metab. 2003;285:E16-24.

31. Sheffield-Moore M, Urban RJ. An overview of the endocrinology of skeletal muscle. Trends Endocrinol Metab. 2004; 15:110-5.

32. Jakubiec-Puka A, Ciechomska I, Morga J, Matusiak A. Contents of myosin heavy chains in denervated slow and fast rat leg muscles. Comp Biochem Physiol B Biochem Mol Biol. 1999;122-B:355-62.

33. Sheffield-Moore M. Androgens and the control of skeletal muscle protein synthesis. Ann Med. 2000;32:181-6.

34. Rhea MR, Alvar BA, Burkett LN, Ball SD. A meta-analysis to determine the dose response for strength development. Med Sci Sports Exerc. 2003;35:456-88.

35. Caiozzo VJ, Baker MJ, Huang K, Chou H, Wu YZ, Baldwin KM. Single-fiber myosin heavy chain polymorphism: how many patterns and what proportions? Am J Physiol Regul Integr Comp Physiol. 2003;285:570-80.

36. Trappe S, Trappe T, Gallagher P, Harber M, Alkner B, Tesch P. Human single muscle fiber function witch 84 day bed-rest and resistance exercise. J Physiol. 2004;557:501-13.

37. Munn J, Herbert RD, Hancock MJ, Gandevia SC. Resistance training for strength: effect of number of sets and contraction speed. Med Sci Sports Exerc. 2005;37:1622-6.

38. Kim PL, Staron RS, Phillips SM. Fasted-state skeletal muscle protein synthesis after resistance exercise is altered with training. J Physiol. 2005;568:283-90.

39. Guzmán M, Saborido A, Castro J, Molano F, Megias A. Treatment with anabolic steroids increases the activity of the mitochondrial outer carnitin palmitoyltransferase in rat liver and fast-twitch muscle. Biochem Pharmacol. 1991;41:833-5.

40. Camargo Filho JCS, Vanderlei LCM, Camargo RCT, Francischeti FA, Belangero WD, Dal Pai V. Efeitos do esteróide anabólico nandrolona sobre o músculo sóleo de ratos submetidos a treinamento físico através de natação: estudo histológico, histoquímico e morfométrico. Rev Bras Med Esporte. 2006;12:243-7.

41. Eriksson A, Kadi F, Malm C, Thornell, LE. Skeletal muscle morphology in power-lifters with and without anabolics steroids. Histochem Cell Biol. 2005;124:167-75.

42. Bisschop A, Gayan-Ramirez G, Rollier H, et al. Effects of nandrolone decanoate on respiratory and peripheral muscles in male and female rats. J Appl Physiol. 1997;82:1112-8.

43. Farrell SF, McGinnis MY. Effects of pubertal anabolic-androgenic steroid (AAS) administration on reproductive and aggressive behaviors in male rats. Behav Neurosci. 2003;117:904-11.

44. Short KR, Vittone JL, Bigelow ML, et al. Changes in myosin heavy chain mRNA and protein expression in human skeletal muscle with age and endurance exercise. J Appl Physiol. 2005;99:95-102.

45. Pette D. Plasticity in skeletal, cardiac and smooth muscle. Historical perspectives: plasticity of mammalian skeletal muscle. J Appl Physiol. 2001;90:1119-24. 
Verlengia R, et al.

\section{Agradecimentos}

Os autores agradecem a Fundação de Amparo a Pesquisa do Estado de São Paulo (FAPESP - 04/06643-1); Fundo de Apoio a Pesquisa UNIMEP-Piracicaba (FAP/UNIMEP) e Conselho Nacional de Desenvolvimento Científico e Tecnológico (CNPq) pela bolsa PROSUP e ao Prof. Dr. Eduardo Caldas Costa pela revisão científica realizada no manuscrito.

ENDEREÇO

Rozangela Verlengia

Grupo de Pesquisa em Ciências da Performance Humana

Faculdade de Ciências da Saúde

Universidade Metodista de Piracicaba

Recebido para publicação: 15/04/2012

Aceito: 29/11/2012

Rod. do Açúcar, km 156 - Campus Taquaral

13423-170 - Piracicaba - SP - BRASIL

e-mail: rverleng@unimep.br

90 • Rev Bras Educ Fís Esporte, (São Paulo) 2013 Jan-Mar;27(1):83-90 